\title{
Efeito redutor do Viagra (Citrato de Sildenafila) sobre a frequência de tumores epiteliais induzidos pela Doxorrubicina em Drosophila melanogaster
}

\author{
Viagra (Sildenafil citrate) reduced the frequency of epithelial \\ tumors induced by doxorubicin in Drosophila melanogaster
}

Samuel Campos Silva ${ }^{1}$, Juliene César dos Santos ${ }^{1}$, Priscila Capelari Orsolin ${ }^{2}$

\begin{abstract}
RESUMO
O consumo de medicamentos está entre as principais causas de desenvolvimento do câncer. Faz-se, então, necessário investigar os efeitos de drogas amplamente consumidas no mundo, como é o caso do Viagra ${ }^{\circledR}$ (Citrato de Sildenafila - CS). O objetivo do presente estudo foi avaliar o efeito modulador do CS na carcinogênese induzida pela Doxorrubicina (DXR), por meio do Teste para Detecção de Clones de Tumores Epiteliais em Drosophila melanogaster. Foram utilizadas duas linhagens mutantes do organismo teste ( $w t s$ e $m w h$ ) no intuito de obter larvas heterozigotas $w t s+/+m w h$, que foram tratadas com três concentrações de CS: 10 mM, 20 mM e 40 mM, isoladamente e em associação com a DXR. Constatou-se que o CS não exerceu efeito carcinogênico nas concentrações isoladamente avaliadas. Quando em associação com a DXR, propiciou efeito redutor sobre as frequências dos tumores induzidos por ela, conferindo papel anticarcinogênico ao CS. Acredita-se que este efeito seja devido a uma supra-regulação da via de apoptose dependente de caspase e ao impedimento do crescimento celular promovido pelo fármaco utilizado. As evidências obtidas comprovam o efeito protetor tumoral atribuído ao Viagra $\mathbb{R}$ nas condições experimentais propostas.
\end{abstract}

Palavras-chave: Viagra. Citrato de Sildenafila. Doxorrubicina. Câncer. Anticarcinógenos. Drosophila melanogaster.

\begin{abstract}
Drug consumption is among the leading causes of cancer development. Therefore, it is necessary to investigate the effects of drugs widely consumed in the world, as the case of Viagra ${ }^{\circledR}$ (Sildenafil Citrate - SC). The objective of the study was to evaluate the modulatory effect of SC on the carcinogenicity induced by Doxorubicin (DXR) by the Test for Detection of Epithelial Tumor Clones in Drosophila melanogaster. Two mutant lines (wts and $m w h$ ) were used in order to obtain heterozygous $w t s+/+m w h$ larvae, which were treated with three concentrations of CS: $10 \mathrm{mM}, 20 \mathrm{mM}$ and $40 \mathrm{mM}$, alone and in combination with doxorubicin. We verified that SC had no carcinogenic effect at the concentrations
\end{abstract}

1. Acadêmico(a) do curso de Medicina, Centro Universitário de Patos de Minas (UNIPAM).

2. Doutora em Genética e Bioquímica, Centro Universitário de Patos de Minas (UNIPAM).
CORRESPONDÊNCIA: Samuel Campos Silva Centro Universitário de Patos de Minas (UNIPAM). Rua Major Gote, 808 - Caiçaras, 38700-207. Patos de Minas / MG.

Recebido em 11/08/2017 Aprovado em 27/02/2018 
evaluated alone. In association with DXR, it was provided a reduced effect of tumor-induced, conferring anticarcinogenic role to SC. It is believed that this effect is due to up-regulation of the caspase-dependent apoptosis and the impediment of cell growth promoted by the drug used. Evidence obtained confirms the tumor protective effect attributed to Viagra ${ }^{\circledR}$ under experimental conditions.

Keywords: Viagra. Sildenafil Citrate. Doxorubicin. Cancer. Anticarcinogens. Drosophila melanogaster.

\section{Introdução}

O corpo humano é inteiramente formado por células que se organizam em tecidos e órgãos. As células habitualmente se dividem, amadurecem e morrem, renovando-se a cada etapa. Todavia, algumas células podem deixar de seguir esse processo natural e, porventura, desenvolverem o câncer. ${ }^{1}$

Câncer é um termo abrangente e descreve mais de 200 doenças que são provocadas por células que se proliferam sem um controle adequado. ${ }^{2}$ É sabido que independentemente da exposição a um agente cancerígeno, as células sofrem processos de mutações espontâneas em seus genes, mas sendo constantemente reparados por mecanismos específicos de controle. Estas afeç̧ões podem ocorrer em genes específicos, os proto-oncogenes, que, quando ativados, transformam-se em oncogenes, os responsáveis pela malignização celular. ${ }^{3}$

A carcinogênese é influenciada pela exposição a diferentes agentes cancerígenos, que são os responsáveis, muitas vezes, pelo início, pela promoção e pela progressão do tumor. Todavia, a presença desses agentes, por si só, pode não ser inteiramente responsável pelo desenvolvimento da doença. ${ }^{4}$ Sabe-se que em vários tipos de cânceres a suscetibilidade genética tem papel importante, mas é a interação entre a suscetibilidade e os fatores ou as condições resultantes do modo de vida e do ambiente que determinam o risco do adoecimento. ${ }^{5}$

O consumo de medicamentos está entre as principais causas apontadas para o surgimento do câncer, ${ }^{6}$ logo, existe uma crescente preocupação em relação ao risco de uso dessas substâncias. ${ }^{7} \mathrm{E}$, apesar das diversas pesquisas e avanços na área oncológica e genética, nem todas os compostos conhecidos foram testados para avaliar seu potencial carcinogênico e/ou anticarcinogênico. Um exemplo disso é o citrato de sildenafila (citrato de $1-[[3-(6,7-$ diidro-1-metil-7-oxo-3-propil-1H-pirazol- [4,3d]pirimidin-5-il) -4-etóxifenil] sulfonil]- 4- metilpi- perazina1), princípio ativo do medicamento Viagra $\AA$, aprovado em 1998 pelo Food and Drug Administration para tratamento da Disfunção Erétil. ${ }^{8}$ Este composto pertence a classe dos inibidores da fostodiesterase, sendo altamente seletivo e potente para a fosfodiesterase tipo 5 , responsável pela degradação do cGMP. ${ }^{9}$ Trata-se de um dos medicamentos mais vendidos em todo o mundo, atingindo mais de 6 milhões de prescrições nos primeiros 6 meses de mercado e gerando bilhões de dólares no comércio farmacêutico. ${ }^{8}$

Estudos recentes evidenciam uma associação positiva entre o uso do Citrato de Sildenafila e o aumento da frequência de apoptoses, bem como a redução da dimensão de tumores e a potencialização do efeito de drogas quimioterápicas. ${ }^{10}$ Diante do exposto, torna-se relevante uma avaliação que busque investigar a relação entre o Citrato de Sildenafila (CS) e seu efeito sobre a carcinogênese. A escolha da Drosophila melanogaster como organismo teste é justificado tanto pela facilidade de manuseio dela, quanto pela similaridade genética com o organismo humano, já que $70 \%$ dos genes oncogênicos no homem possuem similaridade com os genes desta mosca. ${ }^{11}$

Nesse contexto, o presente estudo foi desenvolvido com o objetivo de investigar o efeito modulador do CS na carcinogênese induzida pela Doxorrubicina (DXR) por meio do Teste para Detecção de Clones de Tumores Epiteliais em Drosophila melanogaster ( $D$. melanogaster).

\section{Metodologia}

\section{Agentes químicos}

\section{Citrato de Sildenafila}

O Citrato de Sildenafila é vendido sob o nome comercial de Viagra ${ }^{\circledR}$. É fabricado e embalado por Actavis Italy S.p.A., Nerviano, Milão - Itália. É re- 
gistrado, importado e embalado por laboratórios Pfizer Ltda. As concentrações utilizadas no presente estudo foram: $10 \mathrm{mM}, 20 \mathrm{mM}$ e $40 \mathrm{mM}$. A definição das concentrações foi baseada em estudo desenvolvido por Das et al. (2010).12

\section{Cloridrato de Doxorrubicina}

O cloridrato de doxorrubicina (DXR) é vendi-

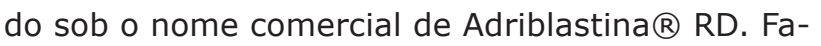
bricado e embalado por Actavis Italy S.p.A., Nerviano, Milão - Itália; registrado e importado por LABORATÓRIOS PFIZER LTDA. Foi utilizado como controle positivo neste estudo, com concentração de $0,4 \mathrm{mM}$ por possuir efeito carcinogênico comprovado. ${ }^{13,14}$

\section{Teste para detecção de tumores epiteliais em drosophila melanogaster}

O teste para detecção de tumor epitelial em Drosophila melanogaster é usado para testar a atividade carcinogênica e antitumoral de substâncias puras ou misturas complexas. ${ }^{15} \mathrm{O}$ gene warts (wts), localizado no cromossomo 3R100A5, foi identificado por Nishiyama et al. (1999) ${ }^{16}$ e codifica uma proteína serina / treonina quinase que atua como supressor de tumor em Drosophila. Sua deleção leva à perda de controle sobre a quantidade e direção da proliferação celular, tornando-o importante no controle da morfogênese. ${ }^{17}$ Homologias entre o wts em Drosophila com o gene LATS1 em mamíferos, localizado cromossomo 6q24-25, já foram descritas, ${ }^{18}$ de forma que deficiências neste gene estão associadas ao desenvolvimento de tumores ovarianos e sarcomas em tecidos moles. ${ }^{16}$

De acordo com Eeken et al. (2002), ${ }^{19}$ a inativação do gene wts nas duas copias do cromossomo na Drosophila resulta em letalidade embrionária. Portanto, o alelo é encontrado na linhagem estoque com a presença do balanceador cromossômico TM3. Larvas heterozigotas são obtidas através do cruzamento de linhagens wts/TM3, Sb1 com a linhagem multiple wing hairs ( $m w h / m w h)$. Entretanto, as células perdem a heterozigose formando clones homozigotos, sendo estes agora viáveis quando encontram-se na larva em conjuntos de células isoladas. Os clones homozigotos manifestam-se então na mosca adulta em forma de tumores. ${ }^{20}$

A linhagem wts foi gentilmente cedida pelo Bloomington Drosophila Stock Center, da Universi- dade de Indiana nos Estados Unidos (USA), registrado sob o número: Bloomington/7052. Já a linhagem $m w h / m w h$ foi cedida pelo Dr. Ulrich Graf (Physiology and Animal Husbandry, Institute of Animal Science, ETH Zurich, Schwerzenbach, Switzerland).

Os estoques das linhagens são cultivados no Laboratório de Citogenética e Mutagênese do Centro Universitário de Patos de Minas - UNIPAM, mantidas em frascos de $1 / 4$ de litro contendo meio de cultura de $D$. melanogaster. Esse meio é composto por $820 \mathrm{~mL}$ de água; $25 \mathrm{~g}$ de fermento (Sacchoromyces cerevisae); $11 \mathrm{~g}$ de ágar; $156 \mathrm{~g}$ de banana e $1 \mathrm{~g}$ de nipagin. As linhagens foram conservadas dentro de uma incubadora B.O.D. $411 \mathrm{D}$, a uma temperatura em torno de $25^{\circ} \mathrm{C}$ e $60 \%$ de umidade.

Para a realização dos cruzamentos, os machos e as fêmeas foram colocados juntos em frascos contendo meio de cultura próprio para postura, onde as fêmeas depositaram seus ovos. Para obtenção de larvas heterozigotas de 72 horas $w t s+/$ + mwh realizou-se o cruzamento entre fêmeas virgens wts/TM3,Sb1 com machos mwh/mwh. As larvas descendentes deste cruzamento foram, então, tratadas com Viagra ${ }^{\circledR}$ em três diferentes concentrações: 10 mM, $20 \mathrm{mM}$ e $40 \mathrm{mM}$. Somente as moscas adultas com características de pelos longos e finos foram analisadas, ou seja, somente as moscas que não são portadoras do balanceador cromossômico TM3, Sb1, uma vez que possuem o gene em estudo.

\section{Procedimento experimental}

A coleta dos ovos descendentes dos cruzamentos entre fêmeas virgens wts/TM3,Sb1 com machos $m w h / m w h$, ocorreu durante um período de 8 horas, em frascos contendo meio de cultura próprio para postura, uma base sólida de ágar ( $3 \%$ de ágar em água) e uma camada de fermento biológico (Saccharomyces cerevisiae) suplementado com sacarose.

Para o tratamento foram utilizadas as larvas de 72 horas resultantes do cruzamento descrito, sendo essas transferidas posteriormente para frascos contendo $1,5 \mathrm{~g}$ de purê de batata (meio alternativo para a $D$. melanogaster). Aproximadamente $50 \%$ das larvas foram tratadas isoladamente com as concentrações associadas de Viagra ${ }^{\circledR}(10,20 \mathrm{e}$ $40 \mathrm{mM}$ ), controle negativo e positivo. Outras larvas 
( $50 \%)$ foram submetidas ao cotratamento com Doxorrubicina. Junto a $0,03538 \mathrm{~g}$ desse composto foram colocados $25 \mathrm{~mL}$ de água (osmose reversa) utilizada como solvente para obter a concentração de 0,4 mM de Doxorrubicina. Posteriormente, a mesma massa de DXR foi diluída separadamente com as três concentrações diferentes de CS, obtendo as concentrações associadas (DXR+ Viagra $\AA$ ) utilizadas na análise do efeito modulador.

Para o controle positivo, foi utilizada Doxorrubicina a 0,4 $\mathrm{mM}$, e a água osmose reversa para o controle negativo. Nesta etapa do tratamento as larvas ficaram expostas aos agentes químicos testados por um período de aproximadamente 48 horas, até ocorrer a empupação.

Após o tratamento, as moscas foram coletadas e armazenadas em frascos devidamente identificados, contendo etanol $\left(\mathrm{C}_{2} \mathrm{H}_{6} \mathrm{O}\right) 70 \%$. Posteriormente, elas foram colocadas individualmente numa placa escavada contendo glicerina (Glicerol $\mathrm{C}_{3} \mathrm{H}_{8} \mathrm{O}_{3}$ ) e analisadas em lupa estereoscópica para visualização e contagem da presença de tumores, de acordo com a descrição de Justice (1995), ${ }^{15}$ com auxílio de um pincel para manusear as moscas.

Para registrar a frequência de tumores foi utilizada uma planilha padrão, que separa quantitativamente a ocorrência de tumores nas regiões do olho, cabeça, asa, corpo, perna, halteres e o total por mosca, em cada concentração testada.

\section{Análise estatística}

As diferenças estatísticas entre as frequências de tumores das concentrações testadas e os controles (positivo e negativo) foram calculadas utilizando o teste $U$, não paramétrico, de Mann-Whitney, empregando o nível de significância $p<0,05$.

\section{Resultados e Discussão}

A partir da Tabela 1 é possível verificar a frequência de tumores epiteliais nos diferentes segmentos do corpo da $D$. melanogaster quando tratadas com os controles (positivo e negativo) e as diferentes concentrações testadas de Viagra $®$. Observa-se que CS exerceu efeito anticarcinogênico/ modulador nas concentrações testadas $(10$ mM, 20 $\mathrm{mM}$ e $40 \mathrm{mM}$ ), uma vez que nas três proporções avaliadas houve redução significativa $(p<0,05)$ na frequência total de tumores quando comparado ao controle positivo isolado (DXR 0,4 mM).

Naqueles organismos tratados com a associação de CS (em concentrações crescentes) e DXR foram verificadas, respectivamente, frequências totais de 0,$28 ; 0,25$ e 0,21 tumores por mosca e, no controle positivo, frequência de 3,76 tumores por mosca. Evidencia-se, portanto, que a substância avaliada foi capaz de atuar na redução de tumores induzidos pela DXR, modulando seu efeito.

Tabela 1. Frequência de clones de tumores observados em Drosophila melanogaster, heterozigota para o gene supressor de tumor wts, tratadas com Doxorrubicina (DXR) e diferentes concentrações de Citrato de Sildenafila (CS).

\begin{tabular}{|c|c|c|c|c|c|c|c|c|c|c|}
\hline \multicolumn{2}{|c|}{ Tratamentos } & \multirow{2}{*}{$\begin{array}{l}\mathrm{N}^{\circ} \mathrm{de} \\
\text { moscas }\end{array}$} & \multicolumn{7}{|c|}{ Número de tumores analisados } & \multirow{2}{*}{$\begin{array}{c}\text { Frequência } \\
\left(\mathrm{N}^{\circ} \text { de }\right. \\
\text { tumores/mosca) }\end{array}$} \\
\hline$(\mathrm{mM})$ & $\begin{array}{l}\text { DXR } \\
(\mathrm{mM})\end{array}$ & & Olho & Cabeça & Asa & Corpo & Perna & Halter & Total & \\
\hline 0 & 0 & 200 & 1 & 3 & 8 & 2 & 1 & 1 & 16 & 0,08 \\
\hline 10 & 0 & 200 & 0 & 2 & 13 & 6 & 2 & 0 & 23 & 0,11 \\
\hline 20 & 0 & 200 & 2 & 0 & 8 & 11 & 2 & 1 & 24 & 0,12 \\
\hline 40 & 0 & 200 & 2 & 1 & 10 & 6 & 2 & 1 & 22 & 0,11 \\
\hline 0 & 0,4 & 200 & 3 & 47 & 482 & 168 & 34 & 18 & 752 & $3,76 *$ \\
\hline 10 & 0,4 & 200 & 0 & 5 & 17 & 26 & 5 & 3 & 56 & $0,28 * *$ \\
\hline 20 & 0,4 & 200 & 0 & 5 & 26 & 11 & 8 & 1 & 51 & $0,25 * *$ \\
\hline 40 & 0,4 & 200 & 0 & 3 & 18 & 10 & 11 & 0 & 42 & $0,21 * *$ \\
\hline
\end{tabular}

Diagnóstico estatístico de acordo com o Teste de Mann-Whitney. Nível de significância $p \leq 0,05$

* Valor considerado diferente do controle negativo $(p £ 0,05)$.

** Valor considerado diferente do controle positivo (DXR 0,4 mM) $(p £ 0,05)$.

DXR, Doxorrubicina. 
Constata-se também que o CS não apresentou efeito carcinogênico em nenhuma das concentrações isoladas experimentadas neste estudo, uma vez que as frequências totais de tumores nas concentrações $10 \mathrm{mM}, 20 \mathrm{mM}$ e $40 \mathrm{mM}$ foram, respectivamente, 0,$11 ; 0,12$ e 0,11 , não havendo diferença estatisticamente significativa $(p>0,05)$ quando comparado ao controle negativo (água osmose reversa).

Para os indivíduos tratados somente com o controle negativo evidenciou-se uma frequência de 0,08 tumor por mosca. De acordo com Alves e Nepomuceno (2012), ${ }^{21}$ essa discreta indução de tumores ocorre devido à predisposição genética intrínseca do organismo teste utilizado no experimento. Enquanto isso, no controle positivo isolado constatou-se uma frequência de 3,76 tumores por mosca, demonstrando que a espécie teste é sensível à indução tumoral. A DXR em altas concentrações afeta a síntese de DNA por meio de quebras uni ou bifilamentares, além de promover trocas entre as cromátides irmãs. ${ }^{22,23}$ Este quimioterápico possui, então, efeito carcinogênico e mutagênico, induzindo significativamente a formação de tumores epiteliais nos segmentos do corpo da mosca, como foi evidenciado neste trabalho.

Os mecanismos responsáveis pelos efeitos anticarcinogênicos do CS não foram diretamente analisados no presente estudo, entretanto algumas hipóteses já foram inferidas. Entre os diferentes mecanismos já relatados acerca da modulação do processo de carcinogênese induzida pelo CS, Tiwari e Chen (2013) ${ }^{24}$ descrevem um aumento do número de apoptoses em diferentes tipos de células tumorais a partir do tratamento com inibidores da fosfodiesterase-5 (iPDE-5), classe do fármaco Viagra $\AA$. Postularam que este efeito anticarcinogênico seja devido a via de apoptose dependente de caspases e ao impedimento do crescimento/desenvolvimento celular, que pode estar associado com o aumento concomitante da regulação de vias descendentes através do aumento do monofosfato cíclico de guanosina (GMPc).

Há estudos relatando ainda o aumento da expressão da PDE-5 no câncer de pulmão de células não pequenas, no câncer de bexiga, no adenocarcinoma de cólon e em metástases cerebrais, em comparação com tecidos normais adjacentes. ${ }^{25-28}$ Esses estudos sugerem um papel importante no controle de crescimento destas células quando a
PDE-5 é supra-regulada. Já a combinação de quimioterapia com $i \mathrm{PDE}-5$ foi relatada como promotora da produção de espécies reativas de oxigênio e de apoptose, que permitem um beneficio no tratamento de uma ampla gama de cânceres, como relatado em estudo in vivo de câncer de próstata ${ }^{17} \mathrm{e}$ no câncer de bexiga. ${ }^{26}$

Há também estudo que demonstra o aumento da taxa de sobrevida e a redução do tamanho de tumores em cérebro de ratos a partir da combinação de drogas iPDE-5 associadas à DXR. ${ }^{29}$ Uma das hipóteses para justificar tal fato é de que o fármaco pode aumentar a distribuição e a eficiência da ligação de drogas antineoplásicos com seus receptores, com subsequente aumento da sensibilidade à droga, ${ }^{28}$ devido, em parte, à ação de relaxamento da musculatura lisa vascular que o CS promove. É associado também à substância testada um potencial radiossensibilizador no câncer de pênis, por promover uma vasodilatação local e reduzir o grau de hipóxia celular. ${ }^{30}$

Chavez, Scottcoffield e Hasanrajab (2013), ${ }^{31}$ por sua vez, realizaram um estudo retrospectivo e determinaram que homens tratados com PDE-5 para DE tinham menores chances de ter câncer de próstata. Esta população de homens apresentava significativamente valores pouco elevados de antígeno prostático específico e maior porcentagem de hiperplasia prostática benigna em comparação com os homens não tratados com inibidores PDE-5.

\section{Conclusões}

O Teste para Detecção de Clones de Tumores Epiteliais em $D$. melanogaster permitiu identificar o efeito redutor do Viagra $\AA$ (Citratato de Sidelnafila) sobre a frequência de tumores induzidos pela Doxorrubicina nas presentes condições experimentais. O potencial carcinogênico do CS não foi evidenciado em nenhuma das doses avaliadas. Os dados observados são semelhantes a resultados já descritos na literatura.

Novos estudos com diferentes metodologias são essenciais para a maior compreensão da atuação e repercussão desta substância no organismo humano. Acredita-se que a exploração das propriedades biológicas do CS no homem possa contribuir de forma benéfica para uma maior qualidade de vida e promoção de avanços importantes na medicina oncológica. 


\section{Referências}

1. Schilithz AOC, Lima FCS, Andrade JHV, Oliveira JFP, Santos MO, Rebelo MS, et al. Estimativa 2016 - Incidencia de Câncer no Brasil. Minitério da Saúde - Instituto Nacional de Câncer José Alencar Gomes da Silva (INCA), Rio de Janeiro 2016; 1: 1-126.

2. Denard B, Lee C, Ye J. Doxorubicin blocks proliferation of cancer cells through proteolytic activation of CREB3L1. Elife. 2012; 1: 1-14.

3. Losso GM, Moraes RS, Gentili AC, Messias-Reason IT. Microsatellite instability - MSI markers (BAT26, BAT25, D2S123, D5S346, D17S250) in rectal cancer. Arq Bras Cir Dig. 2012; 25: 240-4

4. Thuler LCS. Abordagens básicas para controle do câncer. Ministério da Saúde - Instituto Nacional de Câncer (INCARJ) 2012; 1: 1-134.

5. Mendonça GAS, Noronha CP, Almeida LM. A Situação do Câncer no Brasil. Ministério da Saúde - Instituto Nacional do Câncer (INCA) 2006; 1: 1-117.

6. Düsman E, Berti AP, Soares LC, Vicentini VEP. The main mutagens and carcinogens agents of human exposure. Rev Bras Biol. 2012; 7: 66-81.

7. Burns GW, Bottino PJ. Genética. $6^{\text {ed }}$. Rio de Janeiro (RJ): Guanabara Koogan; 1991.

8. Amaral AVC, Silva GA, CostaI APA, Coelho CMM, RenzoI $\mathrm{R}$, Laus JL. Sildenafil citrate on retrobulbar and retinal circulation of rabbits. Ciênc Rural. 2014; 44: 1431-6.

9. Golan DE, Tashjian AH, Armstrong EJ, Armstrong AW. Princípios de Farmacologia: A Base Fisiopatológica da Farmacologia. $3^{\text {ed }}$. Rio de Janeiro (RJ): Guanabara, 2014.

10. Griffiths AF, Wessler SR, Carroll SB, Doebley J. Introdução a genética. $10^{\text {ed }}$. Rio de Janeiro (RJ): Guanabara Koogan; 2006.

11. Das A, Durrant D, Mitchell C, Dent P, Batra SK, Kukreja RC. Sildenafil (Viagra) sensitizes prostate cancer cells to doxorubicin-mediated apoptosis through CD95. Oncotarget. 2016; 7: 4399-413.

12. Das A, Durrant D, Mitchell C, Mayton E, Hoke NN, Salloum FN, et al. Sildenafil increases chemotherapeutic efficacy of doxorubicin in prostate cancer and ameliorates cardiac dysfunction. Proc Natl Acad Sci. USA. 2010; 1: 18202-7.

13. Vasconcelos MA, Orsolin PC, Silva-Oliveira RG, Nepomuceno JC, Spanó MA. Assessment of the carcinogenic potential of high intense-sweeteners through the test for detection of epithelial tumor clones (warts) in Drosophila melanogaster. Food and Chemical Toxicology 2017; 101: 1-7.

14. Orsolin PC, Silva-Oliveira RG, Nepomuceno JC. Assessment of the mutagenic, recombinagenic and carcinogenic potential of orlistat in somatic cells of Drosophila melanogaster. Food and Chemical Toxicology 2012; 50: 2598-604.

15. Silva RG. Efeito modulador do ômega-3 sobre a mutagenicidade e carcinogenicidade da Doxorrubicina em células somáticas de Drosophila melanogaster. Uberlândia. Dissertação [Mestrado em Genética e Bioquímica] - Universidade Federal de Uberlândia, 2011.
16. Nishiyama Y, Hirota T, Morisaki T, Hara T, Marumoto T, Iida $\mathrm{S}$, et al. A human homolog of Drosophila warts tumor suppressor, h-warts, localized to mitotic apparatus and specifically phosphorylated during mitosis. FEBS Lett. 1999; 459: 159-65.

17. Justice RW, Zilian O, Woods DF, Noll M, Bryant PJ. The Drosophila tumor suppressor gene warts encodes a homolog of human myotonic dystrophy kinase and is required for the control of cell shape and proliferation. Genes Dev. 1995; 9: 534-46.

18. Xia H, Qi H, Li Y, Pei J, Barton J, Blackstad M, et al. LATS1 tumor suppressor regulates G2/M transition and apoptosis. Oncogene. $2002 ;$ 21(8):1233-41.

19. Eeken JC, Klink I, van Veen BL, Pastink A, Ferro W. Induction of epithelial tumors in Drosophila melanogaster heterozygous for the tumor suppressor gene wts. Environ Mol Mutagen. 2002; 40:277-82.

20. Sidorov RA, Ugnivenko EG, Khovanova EM, Belitsky GA. Induction of tumor clones in D. melanogaster wts/+ heterozygotes with chemical carcinogens. Mutat Res. 2001; 498:181-91.

21. Alves EM, Nepomuceno JC. Avaliação do efeito anticarcinogênico do látex do avelós (Euphorbia tirucalli), por meio do teste para detecção de clones de tumor (warts) em Drosophila melanogaster. Perquirere 2012; 9: 125-40.

22. Brunton LL, Lazo JS, Parker KL. Goodman e Gilman: As bases farmacológicas da terapêutica. $9^{\text {ed }}$. Rio de Janeiro (RJ): Guanabara Koogan; 1996.

23. Rang HP, Dale MM, Ritter JM. Rang e Dale: Farmacologia. $6^{\text {ed }}$. Rio de Janeiro (RJ): Elsevier; 2007.

24. Tiwari A, Chen ZS. Repurposing phosphodiesterase-5 inhibitors as chemoadjuvants. Frontiers in Pharmacology 2013; 4: 1-3.

25. Piazza GA, Thompson WJ, Pamukcu R, Alila HW, Whitehead CM, Liu L, et al. Exisulind, a novel proapoptotic drug, inhibits rat urinary bladder tumorigenesis. Cancer Res. 2001; 61: 3961-8.

26. Pusztai L, Zhen JH, Arun B, Rivera E, Whitehead C, Thompson WJ, et al. Phase I and II study of exisulind in combination with capecitabine in patients with metastatic breast cancer. J Clin Oncol. 2003; 21: 3454-61.

27. Whitehead CM, Earle KA, Fetter J, Xu S, Hartman T, Chan DC, et al. Exisulind-induced apoptosis in a non-small cell lung cancer orthotopic lung tumor model augments docetaxel treatment and contributes to increased survival. Mol Cancer Ther. 2003; 2: 479-88.

28. Spiess PE. Prostate Cancer - Diagnostic and Therapeutic Advances. $1^{\text {ed }}$. Rijeka (Croácia): InTech, 2011.

29. Black KL, Yin $\mathrm{D}$, Ong JM, Hu J, Konda BM, Wang $X$, et al. PDE5 Inhibitors enhance tumor permeability and efficacy of chemotherapy in a rat brain tumor model. Brain Research 2008; 1230: 290-302.

30. Huilgol NG, Jain A. A new indication of sildenafil in medicine: hypoxic cell sensitizer for penile cancer. J Cancer Res Ther. 2006; 2: 132-5.

31. Chavez AH, Coffield KS, Rajab MH, Jo C. Incidence rate of prostate cancer in mentreated for erectile dysfunction with phosphodiesterase type 5 inhibitors: retrospective analysis. Asian J Androl. 2013; 15: 246-8. 